\title{
Design and Performance Evaluation of High Efficient TCP for HBDP Networks
}

\author{
TaeJoon Park ${ }^{1}$, ManKyu Park ${ }^{2}$, JaeYong Lee ${ }^{2, \star}$, and ByungChul Kim² \\ ${ }^{1}$ Electronics and Telecommunications Research Institute \\ 161 Gajong-Dong, Yuseong-Gu, Daejeon, 305-350, Korea \\ tjpark@etri.re.kr \\ 2 Department of Infocom Engineering, Chungnam National University \\ 220 Gung-Dong, Yuseong-Gu, Daejeon, 305-764, Korea \\ mkpark@ngn.cnu.ac.kr, \{jyl, byckim\}@cnu.ac.kr
}

\begin{abstract}
While the legacy TCP is the most commonly used and reliable transport protocol in the Internet, it is not suitable for massive data transfers in high bandwidth delay product networks. To cope with this problem, we propose high efficient TCP congestion control mechanism that can provide efficient data transfer in HBDP networks. When there is some available bandwidth and it satisfies a certain condition, the congestion window grows rapidly. Otherwise, it maintains a linear growth of the congestion avoidance phase similar to the legacy TCP congestion avoidance algorithm. Based on the relationship of the current and minimum round trip time, the proposed method selects between the linear and rapid growth phases of the congestion window update during the congestion avoidance period. To prevent packet loss during the exponential growth phase, proposed method uses not only the end-to-end delay information, but also the estimated bandwidth of the bottleneck node.
\end{abstract}

Keyword: TCP, and High Bandwidth Delay Product Networks.

\section{Introduction}

As TCP was designed to the reliability guaranteeing transfer protocol, it is most widely used in various network environments as the general transfer protocol. While the transmission control protocol(TCP) is the most commonly used reliable transport protocol, it is generally accepted that standard TCP is not suitable for bulk data transfers in high bandwidth delay product(HBDP) networks. Since the TCP congestion avoidance algorithm is not so dynamic, the packet drop rate needed to fill a Gigabit pipe using the current TCP protocol is beyond the limit of currently achievable fiber optic error rates. Therefore, many TCP congestion control mechanisms are proposed to solve the low efficiency problem in HBDP networks. However, it still has some problems as to the bandwidth efficiency, the RTT fairness convergence time, and etc.

\footnotetext{
* Corresponding author.
} 
We propose a modified congestion avoidance mechanism, HE-TCP, which controls the slow start threshold(ssthresh) with the relationship of current and last available bandwidth in HBDP networks : when the congestion window size is lesser than the current ssthresh size, the congestion window grows aggressively, and otherwise, it maintains linear increase similar to the legacy congestion avoidance algorithm. So, in spite of the compatibility about the legacy TCP, it does not have a meaning to classify according to slow start and congestion avoidance period. The proposed algorithm prevents large packet losses by adjusting congestion window size appropriately. Also, it can rapidly utilize the large available bandwidth by maintaining the ssthresh dynamically. The simulation results show that the proposed algorithm improves not only the utilization of the available bandwidth but also RTT fairness convergence time in high bandwidth delay product network environment.

The rest of the paper is organized as following: Section 2 describes the previous work in this area. Section 3 discusses the proposed mechanism and section 4 describes the simulation results followed by conclusions in section 5

\section{Related Work}

During the initial start up phase (slow start) of the traditional TCP, compliant to the RFC 2581 [1] IETF standards, exponentially increases the amount of transferred data until detecting a packet loss by a timeout or triple duplicate ACKs. When a loss is detected, the TCP halves the congestion window cwnd, which controls the number of packets to be sent without acknowledgement, and moves into the congestion avoidance phase. During the congestion avoidance phase, the TCP increases the cwnd by one packet per the cwnd number of received ACKs and halves the cwnd for a packet drop. Thus, we call the TCP's congestion control algorithms Additive Increase Multiplicative Decrease (AIMD) algorithms. However, the AIMD control of the traditional TCP is not dynamic enough to fill a big pipe for HBDP networks. For example, a standard TCP connection with 1500-byte packets, a $100 \mathrm{~ms}$ RTT and a steady-state throughput of 10 Gbps requires an average congestion window of 83,333 segments and a maximum packet drop rate of one congestion event for every 5,000,000,000 packets 2. The average bit error rate of $\left(2 \times 10^{-14}\right)$ is needed to fully utilize the link in this environment; however it is almost impossible to realize the requirement for the current network technology.

Over the past few years, there has been much research efforts to solve the under utilization problem of traditional TCP in HBDP networks 2 3. High Speed TCP(HS-TCP), recently proposed by Floyd, tries to improve the loss recovery time of standard TCP by changing the increase and decrease parameters of AIMD mechanism. The values for the additive increase range from 1 (standard TCP) to a high of 73 packets, and the range of the multiplicative decrease is from 0.5 (standard TCP) to a low of 0.09. Consequently, when a congestion event occurs over HBDP networks, HS-TCP does not drop back as much and adds more than one packet per RTT, thus recovering faster. However, HS-TCP has some 
drawbacks for deployment in terms of convergence times, compatibility, fairness, and so on [4] [5].

\section{Congestion Control Mechanism of the HE-TCP}

The proposed congestion control mechanism can be divided into three phases: $\mathrm{SS}, \mathrm{CA}$, and RI. The SS phase is the exponential growth phase; the CA phase is the additive increase phase; and the RI phase is the ssthresh controlled growth phase only activated in HBDP networks. Figure 1 shows an example evolution of the congestion window of the proposed HE-TCP. The proposed congestion control mechanism has provided a solution to prevent overshooting in the SS phase and a fast bandwidth reserving solution in the CA phase to switch over to the RI phase.

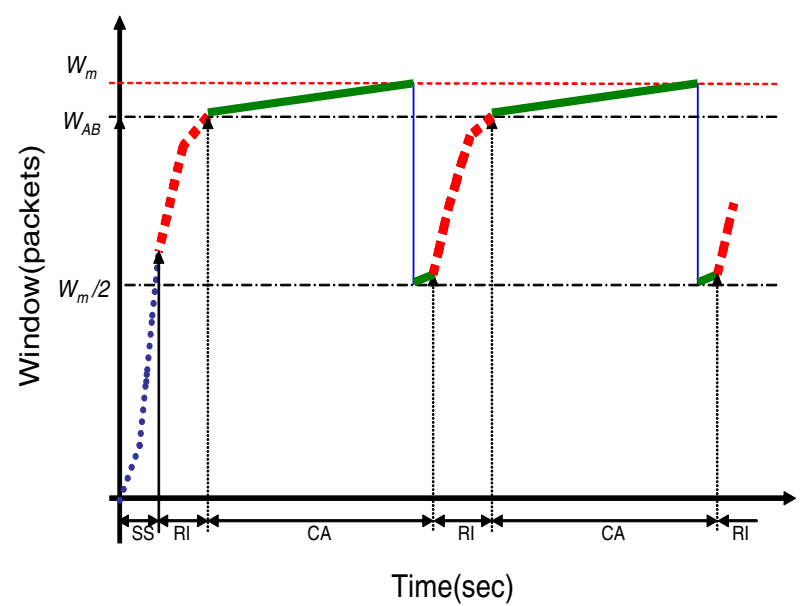

Fig. 1. Evolution example of window size including the startup phase in the proposed HE-TCP

\subsection{Generalized Time-Sliding Window Estimator}

To control the start time of RI, we used a generalized time-sliding window (GTSW) estimator which uses a time-varying coefficient and a exponentiallyweighted moving average filter. The number of computation times per round of GTSW estimator is restricted to a predefined number because of the effect of packets clustering due to congestion and compression. Let $t_{k}$ be the time instant at which the $k_{t h}$ ACK is received at the sender. Let $C u r B W$ and $k$ be the bandwidth share and the time-varying coefficient at time $t_{k}$, respectively. The GTSW estimator is defined as (1):

$$
B W_{k}=\left(1-\alpha_{k}\right) \cdot B W_{k-1}+\alpha_{k} \cdot \operatorname{Cur} B W
$$


where $\alpha_{k}=\Delta t_{k} /\left(R T T_{k}+\Delta t_{k}\right), \Delta t_{k}=t_{k}-t_{k-1}$, and $C u r B W=\left(\sum_{t=t_{k-1}}^{t_{k}} d_{t}\right) / \Delta t_{k}$.

From (11), it follows that

$$
\begin{aligned}
B W_{k} & =\left(1-\frac{\Delta t_{k}}{R T T_{k}+\Delta t_{k}}\right) \cdot B W_{k-1}+\left(\frac{\Delta t_{k}}{R T T_{k}+\Delta t_{k}}\right) \cdot \operatorname{Cur} B W \\
& =\frac{R T T_{k} \cdot B W_{k-1}+\text { PacketSize } \cdot \text { PacketCnt }}{R T T_{k}+\Delta t_{k}}
\end{aligned}
$$

where $R T T_{k}$ is the smoothed round trip time at time $t_{k}$. There have been some previous works on TCP bandwidth measurement methods. From (2), in the case of Available Bandwidth Estimator (ABE) [6], PacketCnt and $\Delta t_{k}$ are cwnd and $R T T$, respectively. Because ABE computes the optimum congestion window once every $R T T$, it is not dynamic enough to estimate the currently usable bandwidth within each round. The PacketCnt and $\Delta t_{k}$ of Time Sliding Window (TSW) 7] Estimator are 1 and inter-packet arrival time, respectively. The TSW Estimator computes and updates the state variables upon each packet arrival which leads to the problem of much computation in HBDP networks. GTSW estimator uses a time-varying coefficient and a exponentially-weighted moving average (EWMA) filter. The GTSW estimator is very similar to the ABE and the TSW Estimator. But The PacketCnt and $\Delta t_{k}$ of TSW Estimator are (cwnd $/ n)$. So the number of computation times per round of GTSW estimator is restricted to predefined number, $n$, because of the effect of packets clustering due to congestion and compression. Therefore, the GTSW estimator is appropriate under the circumstances of HBDP networks. HE-TCP sets the ssthresh to reflect its estimated bandwidth delay product as

$$
\text { SSthresh }=\frac{B W_{k} \cdot \text { BaseRTT }}{\text { PacketSize }}
$$

where BaseRTT is the minimum round trip time, and PacketSize is the packet size.

\subsection{The SS Phase}

The legacy TCP's SS phase is suitable for fast bandwidth reservations. To avoid a premature exit from the SS phase (Fig. 1) and to increase utilization, the initial ssthresh can be increased. However, a large initial ssthresh can cause slow start over-shooting problems and multiple packet losses, and further reduces the utilization. To compensate for the weak points, our mechanism switches the SS phase to the RI phase and limits the maximum size of the cwnd by resetting ssthresh to the estimated available bandwidth of the bottleneck node. Limiting ssthresh to the available bandwidth can limit the fairness in TCPs that use losses for congestion verification. Therefore, in order to estimate the fair share of available bandwidth, bandwidth estimation is computed using a GTSW estimation scheme which has both adaptive gain and adaptive sampling. Usually, most problems in the delay-based congestion control methods, such as 


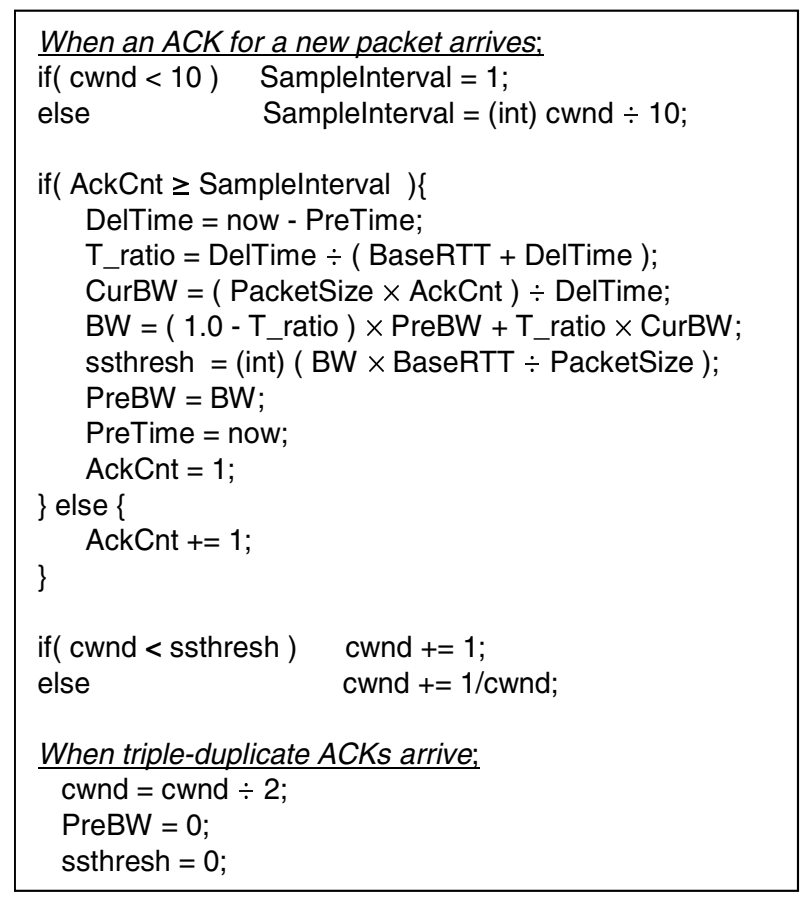

Fig. 2. Pseudo-code for the proposed congestion control

TCP-vegas, are due to the reduction of cwnd depending on the delay. However, the proposed method only decides the cwnd switching point from rapid growth to additive increment and allows bandwidth competition using congestion control, as in the legacy TCP; thus, it minimizes problems in the legacy cwnd control that uses delay-based methods.

\subsection{The CA Phase}

In the TCP Reno, the congestion avoidance phase, CA (Fig. 1), starts when the congestion window exceeds the ssthresh or a packet loss is detected with triple duplicate ACKs. The start condition of the CA phase in the HE-TCP is the same as that of the TCP-reno. However, to manage the under-utilization problem during the CA phase in HBDP networks, we adopt an rapid increase of congestion window in the CA phase, called the RI phase. In the HE-TCP, if the cwnd is smaller than the estimated band-width $B W$, ssthresh, the CA phase switches to the RI phase to drastically increase the congestion window and utilize the larger available bandwidth. If the cwnd exceeds the estimated bandwidth $B W$ during the RI phase, the phase switches back to the CA phase again and the cwnd increases linearly until a packet loss is detected. In the event of a packet loss, the congestion widow size is halved for fairness. Limiting 
ssthresh to the estimated bandwidth, depending on past measurements, can limit the fairness in TCPs that use losses for congestion verification. Therefore, the variables related to the band-width estimation, $B W_{k}$ and $B W_{k-1}$, are initialized to 1 to maintain the fairness.

\subsection{The RI Phase}

The RI phase is similar to the initial slow start phase of the HE-TCP; however, the start time of the RI is controlled by the estimated bandwidth $B W$ of the connection, and yet it is very helpful to solve the fairness problem. As $B W$ approaches to maximum available bandwidth, the increase rate of $c w n d$ is rapidly decreased to make a soft landing avoiding slow start overshoot problems. The exit condition of the RI phase in the HE-TCP is the same as that of the SS phase. Figure 2 shows the pseudo-code of the proposed mechanism. Only when the congestion window is larger than that of the ordinary small BDP networks, the proposed mechanism is used; otherwise, the normal TCP algorithm is used to maintain backward compatibility with the legacy TCP.

\section{Simulation Study}

We performed simulations using the network simulator ns-2 [8] for a network with a bottleneck bandwidth of $800 \mathrm{Mbps}$ and RTT values of $200 \mathrm{~ms}$. The queue size at each bottleneck node is $50 \%$ of the bandwidth delay product of the bottleneck link. For convenience, the window size is measured in number of packets with a packet size of 1000 bytes. Drop tail queues are used in the routers.

Figure 3 shows the congestion window variations of the three TCP variants with a loss rate of $10^{-6}$. In spite of severe packet loss events, the congestion window of the HE-TCP quickly recovers the link capacity and the performance

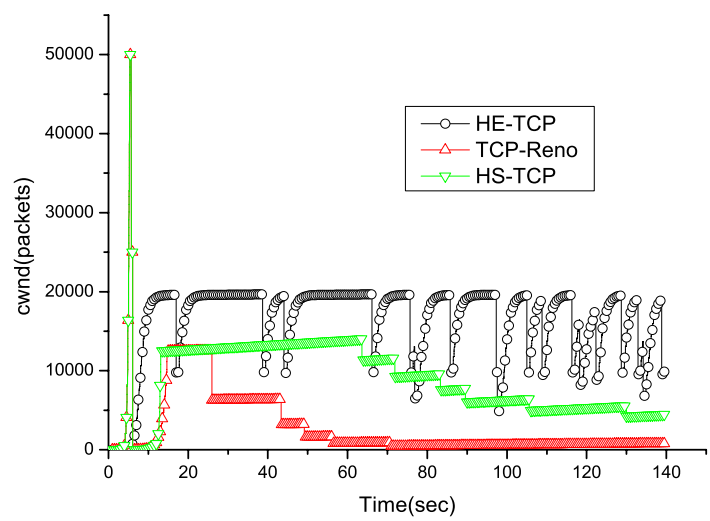

Fig. 3. Congestion window behavior of the HE-TCP, HS-TCP, and TCP Reno mechanisms with a loss rate of $10^{-6}$ 


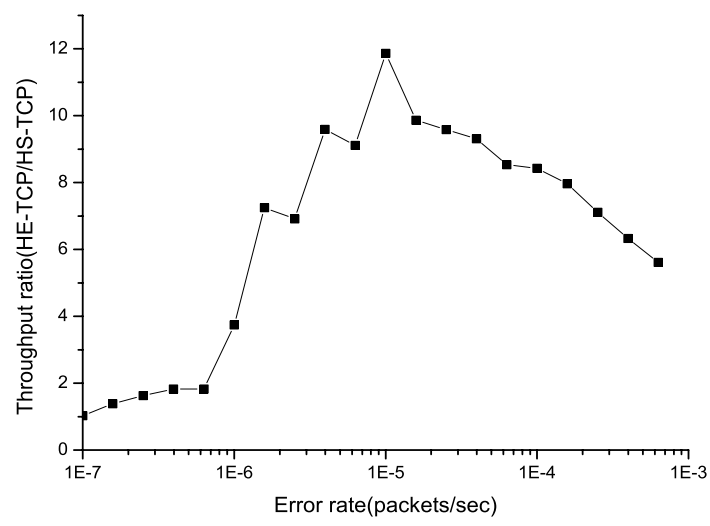

Fig. 4. Throughput ratio of the HE-TCP and HS-TCP mechanisms

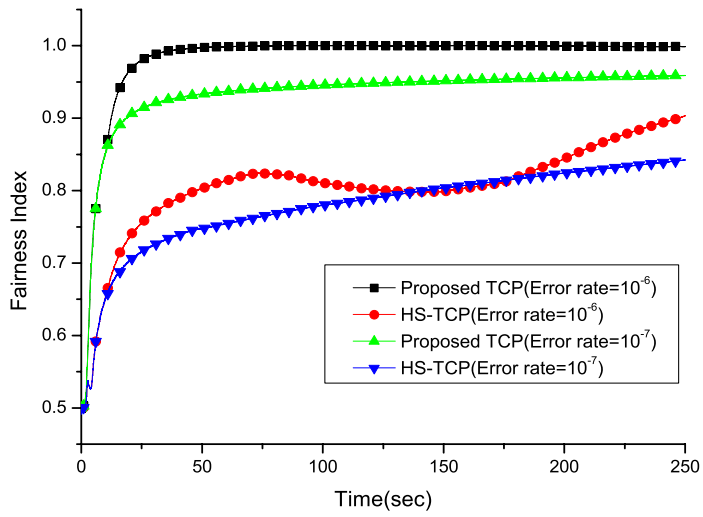

Fig. 5. Fairness comparison of the HE-TCP and HS-TCP mechanisms

is almost maintained at the available bandwidth. However, the performances of the HS-TCP and TCP-Reno are limited by the slow linear increase of the congestion window and the large loss probability.

The performance of the proposed HE-TCP and fairness has been compared with HS-TCP. The parameters for the HS-TCP are set at 31 for low windows, 83,000 for high windows, and $10^{-7}$ for high $\mathrm{p}$.

In Fig. 4 the performance of the HS-TCP is compared with that of the HETCP in terms of packet loss rate. In both cases, the difference is minimal with an almost zero drop rate (less than $10^{-7}$ ), since both use $100 \%$ of the available bandwidth. However, with a reasonably high loss rate, greater than $3 \times 10^{-6}$, the throughput of the HE-TCP is better than that of the HS-TCP.

Figure 5 shows the fairness comparison between the HE-TCP and HS-TCP when the packet loss rate is $10^{-6}$ and $10^{-7}$ with two flows. To show the fair share 
distribution across the connections, we use Jain's Fairness Index as defined in 9. When the throughputs of all flows are equal, the fairness index becomes 1. In both cases, HE-TCP's fairness index converges to 1 faster than the HSTCP. Hence, the HE-TCP shows improved fairness even for cases where the performance difference is minimal due to a low packet loss rate.

\section{Conclusion}

We proposed a modified TCP congestion control mechanism, the HE-TCP, with an rapid increase phase during the congestion avoidance state without overshooting in HBDP networks. In addition, we evaluated the the performance of the proposed HE-TCP.

To evaluate the proposed HE-TCP, it was compared with the HS-TCP, which is the representative TCP for HBDP networks. The simulation results showed that the proposed mechanism improves fairness even when the performance difference is minimal due to a low loss rate. When the loss rate increases, the proposed method was proven to outperform other methods as well. The proposed HE-TCP can solve the TCP under-utilization problem in HBDP networks. The proposed algorithm can be easily implemented in sender TCPs. So, the proposed HE-TCP can be a promising transport protocol for large data transfer applications in HBDP networks.

\section{References}

1. IETF RFC 2581: TCP Congestion Control (1999)

2. IETF RFC 3649: HighSpeed TCP for large congestion windows (2003)

3. Kim, S., Park, S., Moon, J., Lee, H.: A low-crosstalk design of 1.25 Gbps optical triplexer module for FTTH systems. ETRI Journal, Vol. 28, no. 1. (2006) 9-16

4. Wang, R., Pau, G., Yamada, K., Sanadidi, M.Y., Gerla, M.: TCP startup performance in large bandwidth delay networks. Proceeding of IEEE INFOCOM 2004, Vol. 2. (2004) 796-805

5. Mascolo, S., Casetti, C., Gerla, M., Sanadidi, M.Y., Wang, R.: TCP Westwood: Bandwidth estimation for enhanced transport over wireless links. Proceeding of ACM/IEEE Mobi-Com 2001 (2001)

6. Xu, K., Tian, Y., Ansari, N.: TCP-Jersey for Wireless IP Communications. IEEE J. Select. Areas Comulications, Volume 22., (2004) 747-756

7. David D. C., Wenjia F.,: Explicit allocation of best effort packet delivery service. In: IEEE/ACM Trans. Networking, Vol. 6. (1998) 362-373

8. The network simulator ns-2. Available: http://www.isi.edu/nsnam/ns/

9. Jain, R.: The art of computer systems performance analysis: techniques for experimental design, measurement, simulation and modeling. New York, John Wiley \& Sons (1991) 